\title{
ANTIMICROBIAL EFFECT AGAINST ORAL BACTERIA ON BIOACTIVE COMPOUNDS IN A HIGH-PRESSURE ENZYMATIC Prunus mume EXTRACT
}

\author{
EFEITO ANTIMICROBIANO CONTRA BACTÉRIAS ORAIS SOBRE COMPOSTOS \\ BIOATIVOS EM UM EXTRATO ENZIMÁTICO DE Prunus mume DE ALTA PRESSÃO
}

\author{
Sang-Cheol YU ${ }^{1,+}$; So-Ra HAN ${ }^{1,+}$; Jong Hwa JANG ${ }^{2, *}$; Yong-Duk PARK ${ }^{3}$; Tae-Jin OH ${ }^{1,4,5, *}$ \\ 1. Department of Life Science and Biochemical Engineering, SunMoon University, Asansi, Chungnam, Korea; 2. Department of Dental \\ Hygiene, College of Health Science, Dankook University, Cheonan-si, Chungnam, Korea; 3. Professional Graduate School of Dentistry, \\ Kyung Hee University, Seoul, Korea; 4. Department of Pharmaceutical Engineering and Biotechnology, SunMoon University, Asansi, \\ Chungnam, Korea; 5. Genome-based BiolT Convergence Institute, SunMoon University, Asansi, Chungnam, Korea; ‘These authors \\ contributed equally to this work; *Corresponding author, jhjang65@hanmail.net \& tjoh3782@ sunmoon.ac.kr
}

\begin{abstract}
To evaluate high-pressure processing combined with enzymatic treatment for extraction of Prunus mume by determining the optimum extraction conditions for the antimicrobial activity against oral bacteria. Highpressure enzymatic extraction was used to isolate biologically active components from $P$. mume. The effects of process variables such as enzyme type (Pectinex Ultra SP-L, Novozym 33095 and Viscozyme L), enzyme concentration, incubation time/temperature, $\mathrm{pH}$, and ratio of enzymes antimicrobial activity against oral pathogens related to dental caries and periodontal diseases were determined by disk diffusion assay. The optimal conditions for enzymatic extraction from $P$. mume were $\mathrm{pH} 6.0,45^{\circ} \mathrm{C}, 20 \mathrm{~h}$, and $5 \mathrm{v} / \mathrm{v} \%$ with Pectinex Ultra SP-L. The maximum antimicrobial activity of $P$. mume extract obtained using Novozym 33095 was at $\mathrm{pH} 7.0,45^{\circ} \mathrm{C}, 20 \mathrm{~h}$, and $5 \mathrm{v} / \mathrm{v} \%$. The Viscozyme L extract showed the maximum inhibitory effect at $\mathrm{pH} 6.0,45^{\circ} \mathrm{C}, 20 \mathrm{~h}$, and $5 \mathrm{v} / \mathrm{v} \%$. Use of combinations of enzymes did not result in significantly different antimicrobial activity $(p<0.05)$ compared with each enzyme alone. Minimum inhibitory concentration values were 3.125 to $12.50 \%$. These results indicated that high-pressure enzymatic extraction yielded $P$. mume extract with antimicrobial activity which has the potential for improving oral environment.
\end{abstract}

Prunus mume

KEYWORDS: Antimicrobial activity. High-pressure enzymatic extract. Minimum inhibitory concentration.

\section{INTRODUCTION}

The most common oral diseases aredental cavities and periodontal diseases, which are infectious diseases. Dental caries is the medical term for tooth decay or cavities. Gram-positive bacteria are commonly found in the oral cavity and are significant contributors to tooth decay. The most important pathogens of dental caries are mutans streptococci, such as Streptococcus mutans and Streptococcus sobrinus (VAN HOUTE, 1994). Periodontal disease, including gingivitis and periodontitis, is an inflammatory disorder that can destroy periodontal tissue and causes loss of teeth. Aggregatibacter actinomycetemcomitans is the most common pathogen associated with periodontitis, and Actinomyces species are associated with gingivitis (SOCRANSKY; HAFFAJEE, 1992).

Antimicrobial agents for the prevention and treatment of oral disease as alternatives to conventional oral antimicrobial agents, such as chlorhexidine, have been investigated (WALSH et al., 2015). Numerous natural compounds have been reported to be effective in preventing and treating oral infections, and in maintaining good oral health (LIEN et al., 2014). Among them, Prunus mume, known as Japanese apricot, is used as a traditional medicinal food in Korea, China, and Japan. Today, it is cultivated globally for commercial purposes in food and drink because of its health benefits, including anticancer, antioxidant, and antiinflammatory properties (BOUAYED et al., 2009; KAWAHARA et al., 2009). Moreover, P. mume has potential as an oral antimicrobial agent to prevent and treat dental diseases (SENEVIRATNE et al., 2011; JANG et al., 2014). However, the common methods of $P$. mume extraction, including solvent or aqueous thermal-based extraction, result in low yields, long extraction times, and traces of organic solvents (CHO et al., 2013).

Numerous methods have been investigated to enhance plant extraction procedures, such as microwave-assisted extraction, supercritical fluid extraction, ultrahigh-pressure extraction, and enzymatic extraction (JUN et al., 2011). Enzymatic extraction of bioactive compounds from plants is a potential alternative to conventional solvent or aqueous thermal-based extraction. Enzymatic treatment is used in the food industry due to its low operating temperature and ability to increase extraction yields. The enzymes break down plant cell walls, resulting in release of bioactive cell 
contents, including pectic substances, cellulose, hemicelluloses, and lignin (PINELO et al., 2006). High-pressure processing is a novel technology that has shown potential for extraction of bioactive components. High-pressure processing induces structural changes that damage the plant cell wall. Therefore, the bioactive components are more easily washed out, such as flavonoids, alkaloids, vitamins, saponins and pigments. The high-pressure extraction technique is associated with a short extraction time, high extraction yield and lower levels of impurities.

In this study, we evaluated high-pressure processing combined with enzymatic treatment for extraction of $P$. mume and evaluated the antimicrobial activity of the extract against oral bacteria. Several variables-extraction pressure, extraction $\mathrm{pH}$, extraction temperature, extraction time, enzyme concentration, and enzyme ratio-were altered to determine the optimum extraction conditions. In this study, Pectinex Ultra SP-L, Novozym 33095 and Viscozyme L enzymes were used in these extractions. In addition, high-pressure processing was conducted at $100 \mathrm{MPa}$.

\section{MATERIAL AND METHODS}

\section{Materials and reagents}

Prunus mume was obtained from Gwangyang-si, Korea. Pectinex Ultra SP-L, Novozym 33095, and Viscozyme L were purchased from Novozyms Co. (Bagsvaerd, Denmark) and were used to disrupt cell walls. The characteristics of these commercial enzymes are summarized in Table 1.

Table 1. Characteristics of the enzymes used in this study.

\begin{tabular}{|c|c|c|c|}
\hline Commercial name & Enzyme & Source & Activity \\
\hline Pectinex Ultra SP-L & Polygalacturonase & $\begin{array}{l}\text { Aspergillus } \\
\text { aculeatus }\end{array}$ & $3,800 \mathrm{PGNU} / \mathrm{mL}$ \\
\hline Novozym 33095 & $\begin{array}{l}\text { Pectin lyase, } \\
\text { Polygalacturonase }\end{array}$ & $\begin{array}{l}\text { Aspergillus } \\
\text { aculeatus } \\
\text { Aspergillus niger }\end{array}$ & 10,000 PECTU/mL \\
\hline Viscozyme L & $\begin{array}{l}\text { Multi-enzyme complex } \\
\text { containing arabanase, cellulase, } \\
\text { hemicellulase, and xylanase }\end{array}$ & $\begin{array}{l}\text { Aspergillus } \\
\text { aculeatus }\end{array}$ & $100 \mathrm{FBGU} / \mathrm{g}$ \\
\hline
\end{tabular}

PGNU: polygalacturonase unit; PECTU: pectinase unit; FBGU: fungal beta-glucanase unit.

\section{Bacterial strains and growth conditions}

Nine oral bacterial strains were purchased from the Korean Collection for Type Cultures (KCTC, Korea). Staphylococcus aureus ATCC6538P, Streptococcus sobrinus ATCC27607, Streptococcus mutans ATCC25175, Streptococcus ratti ATCC19645, and Streptococcus sanguinis ATCC10556 were grown in Brain-Heart Infusion (BHI; Difco, Detroit, MI, USA) broth. Trypticase Soy Broth (TSB; BD Co., USA) was used for the growth of Streptococcus anginosus ATCC12395, Actinomyces viscosus KCTC5531, Actinomyces naeslundii KCTC5525, and Aggregatibacter actinomycetemcomitans ATCC33384. The bacteria were incubated under anaerobic conditions $\left(85 \% \mathrm{~N}_{2}\right.$, $10 \% \mathrm{H}_{2}$, and $5 \% \mathrm{CO}_{2}$ ) at $37^{\circ} \mathrm{C}$ for $24 \mathrm{~h}$. The bacterial cell density was adjusted to $0.5 \mathrm{McFarland}$ Standard $\left(1.5 \times 10^{8} \mathrm{CFU} / \mathrm{mL}\right)$.

\section{High-pressure enzymatic extraction of $P$. mume}

A reactor (ITC21, Toyokoatsu, Japan) was used for high-pressure enzymatic extraction of $P$. mume. P. mume was extracted using Pectinex Ultra SP-L, Novozym 33095, or Viscozyme L, individually and in combinations. Fifty grams of $P$. mume without seed were added to $150 \mathrm{~mL}$ of buffer ( $\mathrm{pH} 4-8$ ) with $0-10 \mathrm{v} / \mathrm{v} \%$ enzyme. The mixture was placed in a plastic bag, and transferred to a 2.0L stainless steel vessel. High-pressure processing was conducted at $100 \mathrm{MPa}$ for $10-40 \mathrm{~h}$ at $35-$ $65^{\circ} \mathrm{C}$. After incubation, $P$. mume extracts were filtered through filter paper (ADVANTEC No. 2, Advantec MFS, Inc., Tokyo, Japan), concentrated in a rotary evaporator (EYELA A-1000S, Tokyo Rikakikai Co., Japan) and stored at $-20^{\circ} \mathrm{C}$. The highpressure enzyme-aided extraction procedure is described in Figure 1, and the variables are presented in Table 2. 


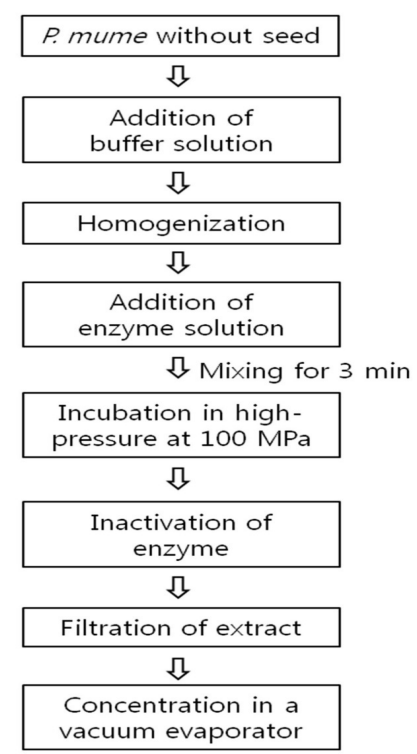

Figure 1. Flow diagram of high-pressure enzyme extraction from P. mume.

Table 2. Extraction variables.

\section{Influence of enzyme concentration}

Changing variables

Enzyme concentration

$0 \mathrm{v} / \mathrm{v} \%$

$1 \mathrm{v} / \mathrm{v} \%$

$5 \mathrm{v} / \mathrm{v} \%$

$10 \mathrm{v} / \mathrm{v} \%$

Constant variables

$\mathrm{pH}$

Temperature

Incubation time

Pressure

Influence of $\mathbf{p H}$

Changing variables

$\mathrm{pH}$

Constant variables

Temperature

Incubation time

Enzyme concentration

Pressure

\section{Influence of temperature}

Changing variables

Temperature

Constant variables

$\mathrm{pH}$

Incubation time

Enzyme concentration

Pressure

Influence of incubation time

Changing variables

Incubation time

Constant variables

$\mathrm{pH}$

Temperature

Enzyme concentration Pressure
$6.0 / 7.0$

$45^{\circ} \mathrm{C}$

$20 \mathrm{~h}$

$100 \mathrm{MPa}$
6.0

7.0

8.0

$45^{\circ} \mathrm{C}$

$20 \mathrm{~h}$

$10 \mathrm{v} / \mathrm{v} \%$

$100 \mathrm{MPa}$

$35^{\circ} \mathrm{C}$

$45^{\circ} \mathrm{C}$

$55^{\circ} \mathrm{C}$

$65^{\circ} \mathrm{C}$

$6.0 / 7.0$

$20 \mathrm{~h}$

$10 \mathrm{v} / \mathrm{v} \%$

$100 \mathrm{MPa}$

$10 \mathrm{~h}$

$20 \mathrm{~h}$

$30 \mathrm{~h}$

$40 \mathrm{~h}$

6.0/7.0

$45^{\circ} \mathrm{C}$

$10 \mathrm{v} / \mathrm{v} \%$

$100 \mathrm{MPa}$ 


\section{Antimicrobial activity against oral bacterial strains}

The antimicrobial activity of $P$. mume extract against nine oral bacterial strains was evaluated by the disc diffusion method (PIDDOCK, 1990). Bacterial suspensions were spread on agar plates using cotton swabs, and sterile filter paper discs (6 mm diameter, Advantec, Japan) soaked in $20 \mu \mathrm{L}$ extract were placed on the preloaded plates. The plates were incubated anaerobically at $37^{\circ} \mathrm{C}$ for $12 \mathrm{~h}$, and then the diameter of the inhibition zones around the discs was measured in millimeters.

\section{Determination of MIC against oral bacterial strains}

The minimum inhibitory concentration (MIC) of $P$. mume extract was determined using the NCCLS reference broth dilution method with slight modifications (SWENSON et al., 1982). This test was performed in sterile, flat-bottom 96-well microtiter plates (F-Type, SPL-Lifesciences Co. Ltd, Korea). P. mume extract was serially diluted twofold to final concentrations of 25 to $0.0997 \%$ of the original extract, and then added to diluted bacterial suspensions $\left(1.0 \times 10^{5} \mathrm{CFU} / \mathrm{mL}\right)$ at a $1: 1$ ratio. After incubation at $37^{\circ} \mathrm{C}$ for $12 \mathrm{~h}$, bacterial growth was measured by determining the absorbance of the suspensions at $600 \mathrm{~nm}$ against a culture medium blank.

\section{Statistical analysis}

All measurements were performed in triplicate and data are presented as means \pm standard deviation of triplicate experiments. Statistical analysis was carried out using PASW Statistics 18.0 (SPSS Inc., Chicago, IL, USA). The significance of differences between values was determined by ANOVA/Duncan's multiple range tests, using a $95 \%$ criterion for a significant difference $(p<0.05)$.

\section{RESULTS AND DISCUSSION}

\section{Optimum high-pressure enzymatic extraction conditions}

Enzymatic extraction was performed using Pectinex Ultra SP-L, Novozym 33095, and Viscozyme L. To determine the optimum enzyme concentration, $P$. mume was extracted at $\mathrm{pH}$ 7.0, $45^{\circ} \mathrm{C}$ and $100 \mathrm{MPa}$ for $20 \mathrm{~h}$ using enzyme concentrations of 0 to $10 \mathrm{v} / \mathrm{v} \%$ according to a previous report (CHO et al., 2013). Then, the antimicrobial activity of the $P$. mume extracts was determined by the disc diffusion method. As shown in Table 3, the optimum concentration of all of
Pectinex Ultra SP-L, Novozym 33095, and Viscozyme L treatment was 5 v/v\%. Antimicrobial activity increased with increasing enzyme concentration up to $5 \mathrm{v} / \mathrm{v} \%$. All Pectinex Ultra SP-L treatment increased the antimicrobial activity from 103 to $168 \%$ compared to non-enzymatic extraction. Viscozyme $\mathrm{L}$ treatment increased antimicrobial activity was 102 to $165 \%$. Novozym 33095 treatment resulted in significant increases in antimicrobial activity of 114 to $231 \%$.

The effect of incubation time $(10-40 \mathrm{~h})$ in the presence of $5 \mathrm{v} / \mathrm{v} \%$ Ultra SP-L, Novozym 33095 and Viscozyme L was next determined. As shown in Table 4, the antimicrobial activities of the extracts increased up to $20 \mathrm{~h}$ and decreased thereafter; however, none of the differences was significant.

Next, the effect of temperature $\left(35-65^{\circ} \mathrm{C}\right)$ in the presence of $5 \mathrm{v} / \mathrm{v} \%$ Pectinex Ultra SP-L, Novozym 33095 and Viscozyme L at $100 \mathrm{MPa}$ for $20 \mathrm{~h}$ on antimicrobial activity was evaluated. The extraction was performed at the optimum $\mathrm{pH}$ value of each enzyme $(\mathrm{pH} 6.0$ for Ultra SP-L and Viscozyme L, pH 7.0 for Novozym 33095). As shown in Table $5,45^{\circ} \mathrm{C}$ was the optimum temperature, irrespective of the enzyme used.

Finally, the optimum $\mathrm{pH}$ value was determined. $P$. mume was extracted using different $\mathrm{pH}$ values ( $\mathrm{pH} 5.0$ to 8.0 for Novozym 33095 and Viscozyme L, and $\mathrm{pH} 4.0$ to 7.0 for Pectinex Ultra SP-L), while temperature $\left(45^{\circ} \mathrm{C}\right)$, enzyme concentration $(5 \mathrm{v} / \mathrm{v} \%)$, pressure $(100 \mathrm{MPa})$ were kept constant for $20 \mathrm{~h}$. As shown in Table 6, $P$. mume extract showed similar antimicrobial activity against all eight bacterial strains. However, the highest antimicrobial activity of the Novozym 33095 extract was at $\mathrm{pH} 7.0$, which resulted in an inhibition zone of 13.76 to $16.53 \mathrm{~mm}$. This extract exhibited the strongest antimicrobial activity against A. naeslundii and the weakest against $S$. anginosus. Pectinex Ultra SP-L and Viscozyme L resulted in zones of inhibition of up to 15.58 and $16.14 \mathrm{~mm}$, respectively, against $A$. actinomycetemcomitans at pH 6.0.

Additionally, compared to Novozym 33095, the effects of combinations of enzymes against $A$. naeslundii and A. actinomycetemcomitans were investigated. As shown in Figure 2, no synergistic effect of enzyme combinations was detected. The combination of Novozym 33095 and Pectinex Ultra SP-L produced an inhibition effect similar to that of each enzyme alone. However, use of Viscozyme L in combination with either of the other two enzymes resulted in a reduced inhibitory effect. 
Table 3. Antimicrobial activities of $P$. mume extract according to enzyme concentration.

\section{Diameter of the zone of inhibition (mm, Means \pm SD)}

\begin{tabular}{|c|c|c|c|c|c|c|c|c|c|c|c|c|}
\hline \multirow[b]{3}{*}{ Microorganisms } & \\
\hline & \multicolumn{4}{|c|}{ Pectinex Ultra SP-L } & \multicolumn{4}{|c|}{ Novozym 33095} & \multicolumn{4}{|c|}{ Viscozyme L } \\
\hline & $0 \%$ & $1 \%$ & $5 \%$ & $10 \%$ & $0 \%$ & $1 \%$ & $5 \%$ & $10 \%$ & $0 \%$ & $1 \%$ & $5 \%$ & $10 \%$ \\
\hline $\begin{array}{l}\text { Staphylococcus } \\
\text { sobrinus }\end{array}$ & $8.29 \pm 0.33^{b}$ & $8.95 \pm 0.21^{\mathrm{b}}$ & $10.46 \pm 0.27^{\mathrm{a}}$ & $9.08 \pm 0.76^{\mathrm{b}}$ & $6.07 \pm 0.07^{\mathrm{c}}$ & $9.28 \pm 0.76^{\mathrm{b}}$ & $11.78 \pm 1.68^{\mathrm{a}}$ & $11.95 \pm 0.16^{\mathrm{a}}$ & $9.23 \pm 0.97^{\mathrm{b}}$ & $9.77 \pm 0.80^{\mathrm{ab}}$ & $11.88 \pm 1.33^{\mathrm{a}}$ & $11.26 \pm 1.89^{\mathrm{ab}}$ \\
\hline $\begin{array}{l}\text { Streptococcus } \\
\text { aureus }\end{array}$ & $7.96 \pm 0.21^{\mathrm{c}}$ & $8.95 \pm 0.37^{\mathrm{b}}$ & $10.46 \pm 0.55^{\mathrm{a}}$ & $9.08 \pm 0.07^{\mathrm{b}}$ & $6.02 \pm 0.01^{\mathrm{c}}$ & $7.86 \pm 0.40^{\mathrm{b}}$ & $11.48 \pm 0.81^{\mathrm{a}}$ & $11.13 \pm 0.90^{\mathrm{a}}$ & $8.29 \pm 0.67^{\mathrm{b}}$ & $8.99 \pm 0.99^{\mathrm{b}}$ & $12.16 \pm 0.38^{\mathrm{a}}$ & $11.82 \pm 0.51^{\mathrm{a}}$ \\
\hline $\begin{array}{l}\text { Streptococcus } \\
\text { mutans }\end{array}$ & $7.32 \pm 0.31^{\mathrm{b}}$ & $7.99 \pm 0.25^{\mathrm{ab}}$ & $8.98 \pm 1.01^{\mathrm{a}}$ & $8.36 \pm 1.07^{\mathrm{ab}}$ & $6.08 \pm 0.09^{c}$ & $8.87 \pm 0.26^{\mathrm{b}}$ & $11.10 \pm 0.75^{\mathrm{a}}$ & $11.46 \pm 0.92^{\mathrm{a}}$ & $8.75 \pm 1.92^{\mathrm{b}}$ & $10.58 \pm 0.97^{\mathrm{ab}}$ & $13.13 \pm 2.13^{\mathrm{a}}$ & $12.04 \pm 2.43^{\mathrm{ab}}$ \\
\hline Streptococcus ratti & $7.55 \pm 0.56^{\mathrm{b}}$ & $8.77 \pm 0.85^{\mathrm{ab}}$ & $9.39 \pm 0.77^{\mathrm{a}}$ & $9.03 \pm 0.30^{\mathrm{a}}$ & $6.02 \pm 0.02^{\mathrm{c}}$ & $9.62 \pm 0.47^{\mathrm{b}}$ & $10.73 \pm 0.40^{\mathrm{a}}$ & $10.42 \pm 0.39^{\mathrm{a}}$ & $7.85 \pm 0.80^{\mathrm{b}}$ & $9.53 \pm 0.85^{\mathrm{a}}$ & $10.65 \pm 0.60^{\mathrm{a}}$ & $9.94 \pm 0.39^{\mathrm{a}}$ \\
\hline $\begin{array}{l}\text { Streptococcus } \\
\text { sanguinis }\end{array}$ & $7.25 \pm 0.82^{\mathrm{a}}$ & $7.50 \pm 1.00^{\mathrm{a}}$ & $8.52 \pm 1.90^{\mathrm{a}}$ & $7.76 \pm 0.95^{\mathrm{a}}$ & $6.01 \pm 0.01^{\mathrm{b}}$ & $6.87 \pm 0.52^{\mathrm{b}}$ & $11.14 \pm 0.84^{\mathrm{a}}$ & $10.28 \pm 0.69^{\mathrm{a}}$ & $8.43 \pm 0.42^{\mathrm{c}}$ & $8.89 \pm 0.39^{\mathrm{bc}}$ & $10.60 \pm 0.63^{\mathrm{a}}$ & $9.95 \pm 1.04^{\mathrm{ab}}$ \\
\hline $\begin{array}{l}\text { Actinomyces } \\
\text { viscosus }\end{array}$ & $7.73 \pm 1.69^{\mathrm{b}}$ & $12.69 \pm 1.85^{\mathrm{a}}$ & $13.00 \pm 1.61^{\mathrm{a}}$ & $12.44 \pm 0.36^{\mathrm{a}}$ & $6.76 \pm 0.54^{c}$ & $12.32 \pm 1.11^{\mathrm{b}}$ & $14.00 \pm 0.70^{\mathrm{a}}$ & $12.96 \pm 0.50^{\mathrm{ab}}$ & $10.53 \pm 1.36^{\mathrm{b}}$ & $14.03 \pm 1.42^{\mathrm{a}}$ & $15.09 \pm 1.88^{\mathrm{a}}$ & $13.82 \pm 1.64^{\mathrm{a}}$ \\
\hline $\begin{array}{l}\text { Actinomyces } \\
\text { naeslundii }\end{array}$ & $9.54 \pm 0.92^{\mathrm{b}}$ & $10.56 \pm 0.57^{\mathrm{ab}}$ & $11.42 \pm 1.00^{\mathrm{a}}$ & $11.77 \pm 0.41^{\mathrm{a}}$ & $6.21 \pm 0.25^{\mathrm{c}}$ & $9.35 \pm 0.68^{b}$ & $14.16 \pm 1.10^{\mathrm{a}}$ & $13.50 \pm 0.49^{\mathrm{a}}$ & $9.68 \pm 0.22^{\mathrm{b}}$ & $13.04 \pm 0.50^{\mathrm{a}}$ & $13.15 \pm 0.88^{\mathrm{a}}$ & $12.06 \pm 0.58^{\mathrm{a}}$ \\
\hline $\begin{array}{l}\text { Streptococcus } \\
\text { anginosus }\end{array}$ & $8.06 \pm 0.42^{b}$ & $8.59 \pm 0.07^{\mathrm{b}}$ & $11.21 \pm 1.50^{\mathrm{a}}$ & $9.65 \pm 0.72^{\mathrm{ab}}$ & $6.01 \pm 0.01^{\mathrm{c}}$ & $9.50 \pm 0.88^{\mathrm{b}}$ & $13.88 \pm 0.36^{\mathrm{a}}$ & $13.00 \pm 0.63^{\mathrm{a}}$ & $9.61 \pm 0.22^{\mathrm{c}}$ & $9.76 \pm 0.50^{\mathrm{bc}}$ & $12.01 \pm 0.88^{\mathrm{a}}$ & $10.87 \pm 0.58^{\mathrm{b}}$ \\
\hline $\begin{array}{l}\text { Aggregatibacter } \\
\text { actinomycetemcomit }\end{array}$ & $8.51 \pm 1.27^{\mathrm{b}}$ & $10.09 \pm 0.48^{\mathrm{b}}$ & $12.89 \pm 0.78^{\mathrm{a}}$ & $12.12 \pm 1.30^{\mathrm{a}}$ & $6.27 \pm 0.05^{\mathrm{c}}$ & $10.77 \pm 1.39^{b}$ & $13.13 \pm 0.48^{\mathrm{a}}$ & $12.16 \pm 1.35^{\mathrm{ab}}$ & $7.75 \pm 1.60^{\mathrm{b}}$ & $11.16 \pm 0.71^{\mathrm{a}}$ & $12.75 \pm 1.82^{\mathrm{a}}$ & $11.56 \pm 2.10^{\mathrm{a}}$ \\
\hline
\end{tabular}
ans

Values represent means \pm standard deviation, $n=3$.

Different letters in rows indicate significant differences between mean values of treatments $(p<0.05)$. 
Table 4. Antimicrobial activities of $P$. mume extract according to incubation time.

\begin{tabular}{|c|c|c|c|c|c|c|c|c|c|c|c|c|}
\hline \multirow[b]{3}{*}{ Microorganisms } & \multicolumn{12}{|c|}{ Diameter of the zone of inhibition $(\mathrm{mm}$, Means $\pm \mathrm{SD})$} \\
\hline & \multicolumn{4}{|c|}{ Pectinex Ultra SP-L } & \multicolumn{4}{|c|}{ Novozym 33095} & \multicolumn{4}{|c|}{ Viscozyme L } \\
\hline & $10 \mathrm{~h}$ & $20 \mathrm{~h}$ & $30 \mathrm{~h}$ & $40 \mathrm{~h}$ & $10 \mathrm{~h}$ & $20 \mathrm{~h}$ & $30 \mathrm{~h}$ & $40 \mathrm{~h}$ & $10 \mathrm{~h}$ & $20 \mathrm{~h}$ & $30 \mathrm{~h}$ & $40 \mathrm{~h}$ \\
\hline $\begin{array}{l}\text { Staphylococcus } \\
\text { sobrinus }\end{array}$ & $13.89 \pm 1.29^{\mathrm{a}}$ & $14.49 \pm 1.28^{\mathrm{a}}$ & $13.9 \pm 1.00^{\mathrm{a}}$ & $11.21 \pm 0.89^{\mathrm{b}}$ & $11.46 \pm 0.95^{\mathrm{a}}$ & $13.62 \pm 2.40^{\mathrm{a}}$ & $12.38 \pm 0.57^{\mathrm{a}}$ & $13.09 \pm 0.47^{\mathrm{a}}$ & $10.55 \pm 0.29^{\mathrm{a}}$ & $12.35 \pm 1.53^{\mathrm{a}}$ & $11.75 \pm 0.85^{\mathrm{a}}$ & $10.52 \pm 0.79^{\mathrm{a}}$ \\
\hline $\begin{array}{l}\text { Streptococcus } \\
\text { aureus }\end{array}$ & $12.68 \pm 1.55^{\mathrm{a}}$ & $14.33 \pm 1.83^{\mathrm{a}}$ & $13.76 \pm 2.20^{\mathrm{a}}$ & $12.58 \pm 1.74^{\mathrm{a}}$ & $11.42 \pm 1.07^{\mathrm{b}}$ & $14.13 \pm 0.31^{\mathrm{ab}}$ & $12.87 \pm 1.50^{\mathrm{ab}}$ & $14.72 \pm 2.10^{\mathrm{a}}$ & $13.90 \pm 0.86^{\mathrm{a}}$ & $14.95 \pm 0.99^{\mathrm{a}}$ & $14.03 \pm 1.95^{\mathrm{a}}$ & $13.45 \pm 1.16^{\mathrm{a}}$ \\
\hline $\begin{array}{l}\text { Streptococcus } \\
\text { mutans }\end{array}$ & $12.65 \pm 0.94^{\mathrm{a}}$ & $13.13 \pm 0.98^{\mathrm{a}}$ & $13.19 \pm 1.09^{\mathrm{a}}$ & $12.58 \pm 1.28^{\mathrm{a}}$ & $11.00 \pm 0.87^{\mathrm{b}}$ & $14.34 \pm 1.06^{\mathrm{a}}$ & $12.57 \pm 1.77^{\mathrm{ab}}$ & $12.51 \pm 2.05^{\mathrm{ab}}$ & $11.24 \pm 0.77^{\mathrm{a}}$ & $12.68 \pm 0.99^{\mathrm{a}}$ & $12.63 \pm 1.86^{\mathrm{a}}$ & $11.71 \pm 1.16^{\mathrm{a}}$ \\
\hline Streptococcus ratti & $13.05 \pm 2.37^{\mathrm{a}}$ & $12.91 \pm 1.22^{\mathrm{a}}$ & $13.17 \pm 1.40^{\mathrm{a}}$ & $12.13 \pm 0.99^{\mathrm{a}}$ & $10.94 \pm 1.16^{\mathrm{b}}$ & $13.60 \pm 0.18^{\mathrm{a}}$ & $12.52 \pm 0.9^{\mathrm{a}}$ & $12.45 \pm 0.52^{\mathrm{a}}$ & $11.82 \pm 2.14^{\mathrm{b}}$ & $15.69 \pm 1.18^{\mathrm{a}}$ & $14.47 \pm 1.89^{\mathrm{ab}}$ & $12.87 \pm 1.67^{\mathrm{at}}$ \\
\hline $\begin{array}{l}\text { Streptococcus } \\
\text { sanguinis }\end{array}$ & $12.37 \pm 0.96^{\mathrm{a}}$ & $12.04 \pm 0.90^{\mathrm{a}}$ & $11.02 \pm 1.06^{\mathrm{a}}$ & $10.85 \pm 0.52^{\mathrm{a}}$ & $10.09 \pm 0.61^{\mathrm{a}}$ & $12.21 \pm 1.25^{\mathrm{a}}$ & $11.89 \pm 0.89^{\mathrm{a}}$ & $12.03 \pm 2.01^{\mathrm{a}}$ & $11.41 \pm 0.11^{\mathrm{c}}$ & $14.00 \pm 0.57^{\mathrm{a}}$ & $13.02 \pm 0.86^{\mathrm{ab}}$ & $12.09 \pm 0.82^{\mathrm{bc}}$ \\
\hline $\begin{array}{l}\text { Actinomyces } \\
\text { viscosus }\end{array}$ & $15.13 \pm 0.14^{\mathrm{a}}$ & $14.15 \pm 1.76^{\mathrm{a}}$ & $15.27 \pm 1.27^{\mathrm{a}}$ & $14.74 \pm 1.07^{\mathrm{a}}$ & $13.31 \pm 0.36^{\mathrm{a}}$ & $15.63 \pm 1.51^{\mathrm{a}}$ & $14.37 \pm 1.54^{\mathrm{a}}$ & $14.65 \pm 1.71^{\mathrm{a}}$ & $11.53 \pm 0.81^{\mathrm{b}}$ & $14.54 \pm 2.58^{\mathrm{a}}$ & $14.04 \pm 1.04^{\mathrm{ab}}$ & $12.43 \pm 0.42^{\mathrm{at}}$ \\
\hline $\begin{array}{l}\text { Actinomyces } \\
\text { naeslundii }\end{array}$ & $14.56 \pm 1.26^{\mathrm{a}}$ & $13.70 \pm 1.53^{\mathrm{a}}$ & $14.27 \pm 0.49^{\mathrm{a}}$ & $13.95 \pm 0.75^{\mathrm{a}}$ & $13.37 \pm 1.27^{\mathrm{a}}$ & $14.87 \pm 1.02^{\mathrm{a}}$ & $13.95 \pm 0.67^{\mathrm{a}}$ & $14.45 \pm 0.09^{\mathrm{a}}$ & $11.71 \pm 1.14^{\mathrm{a}}$ & $13.45 \pm 1.77^{\mathrm{a}}$ & $13.40 \pm 0.84^{\mathrm{a}}$ & $11.71 \pm 0.87^{\mathrm{a}}$ \\
\hline $\begin{array}{l}\text { Streptococcus } \\
\text { anginosus } \\
\text { Aggregatibacter }\end{array}$ & $14.16 \pm 1.53^{\mathrm{a}}$ & $14.18 \pm 2.10^{\mathrm{a}}$ & $13.96 \pm 0.80^{\mathrm{a}}$ & $13.74 \pm 0.78^{\mathrm{a}}$ & $12.38 \pm 0.50^{\mathrm{b}}$ & $14.33 \pm 0.74^{\mathrm{a}}$ & $13.04 \pm 0.87^{\mathrm{b}}$ & $12.62 \pm 0.09^{\mathrm{b}}$ & $11.35 \pm 1.39^{\mathrm{a}}$ & $13.47 \pm 1.24^{\mathrm{a}}$ & $12.98 \pm 1.69^{\mathrm{a}}$ & $11.48 \pm 0.77^{\mathrm{a}}$ \\
\hline actinomycetemcomi & $14.59 \pm 0.79^{\mathrm{a}}$ & $14.20 \pm 1.67^{\mathrm{a}}$ & $14.59 \pm 0.33^{\mathrm{a}}$ & $13.71 \pm 0.57^{\mathrm{a}}$ & $11.76 \pm 2.02^{\mathrm{b}}$ & $15.00 \pm 1.24^{\mathrm{a}}$ & $13.24 \pm 0.48^{\mathrm{ab}}$ & $13.67 \pm 0.98^{\mathrm{ab}}$ & $11.16 \pm 1.85^{\mathrm{a}}$ & $13.58 \pm 1.32^{\mathrm{a}}$ & $13.40 \pm 0.24^{\mathrm{a}}$ & $11.96 \pm 1.29^{\mathrm{a}}$ \\
\hline
\end{tabular}
tans

Values represent means \pm standard deviation, $\mathrm{n}=3$

Different letters in rows indicate significant differences between mean values of treatments $(p<0.05)$. 
Table 5. Antimicrobial activities of P. mume extract according to temperature.

\section{Diameter of the zone of inhibition (mm, Means \pm SD)}

\begin{tabular}{|c|c|c|c|c|c|c|c|c|c|c|c|c|}
\hline \multirow[b]{3}{*}{ Microorganisms } & \\
\hline & \multicolumn{4}{|c|}{ Pectinex Ultra SP-L } & \multicolumn{4}{|c|}{ Novozym 33095} & \multicolumn{4}{|c|}{ Viscozyme L } \\
\hline & $35^{\circ} \mathrm{C}$ & $45^{\circ} \mathrm{C}$ & $55^{\circ} \mathrm{C}$ & $65^{\circ} \mathrm{C}$ & $35^{\circ} \mathrm{C}$ & $45^{\circ} \mathrm{C}$ & $55^{\circ} \mathrm{C}$ & $65^{\circ} \mathrm{C}$ & $35^{\circ} \mathrm{C}$ & $45^{\circ} \mathrm{C}$ & $55^{\circ} \mathrm{C}$ & $65^{\circ} \mathrm{C}$ \\
\hline $\begin{array}{l}\text { Staphylococcus } \\
\text { sobrinus }\end{array}$ & $13.28 \pm 0.67^{\mathrm{a}}$ & $14.23 \pm 1.14^{\mathrm{a}}$ & $13.21 \pm 1.18^{\mathrm{a}}$ & $13.27 \pm 0.63^{\mathrm{a}}$ & $13.45 \pm 1.05^{\mathrm{a}}$ & $14.77 \pm 1.18^{\mathrm{a}}$ & $13.13 \pm 0.50^{\mathrm{a}}$ & $13.44 \pm 1.07^{\mathrm{a}}$ & $13.16 \pm 0.82^{\mathrm{ab}}$ & $14.50 \pm 0.40^{\mathrm{a}}$ & $13.10 \pm 0.83^{\mathrm{ab}}$ & $12.84 \pm 0.88^{\mathrm{b}}$ \\
\hline $\begin{array}{l}\text { Streptococcus } \\
\text { aureus }\end{array}$ & $13.16 \pm 0.70^{\mathrm{ab}}$ & $14.14 \pm 0.47^{\mathrm{a}}$ & $12.99 \pm 1.01^{\mathrm{b}}$ & $13.32 \pm 0.37^{\mathrm{ab}}$ & $13.82 \pm 0.63^{\mathrm{a}}$ & $15.27 \pm 0.77^{\mathrm{a}}$ & $12.77 \pm 1.89^{\mathrm{a}}$ & $13.59 \pm 1.32^{\mathrm{a}}$ & $13.14 \pm 2.60^{\mathrm{a}}$ & $13.85 \pm 2.13^{\mathrm{a}}$ & $13.14 \pm 1.89^{\mathrm{a}}$ & $12.80 \pm 2.35^{\mathrm{a}}$ \\
\hline $\begin{array}{l}\text { Streptococcus } \\
\text { mutans }\end{array}$ & $11.26 \pm 1.63^{\mathrm{a}}$ & $11.63 \pm 1.61^{\mathrm{a}}$ & $11.26 \pm 1.51^{\mathrm{b}}$ & $11.24 \pm 1.27^{\mathrm{a}}$ & $11.77 \pm 0.46^{\mathrm{ab}}$ & $12.90 \pm 0.46^{\mathrm{a}}$ & $10.80 \pm 1.62^{\mathrm{b}}$ & $11.84 \pm 0.36^{\mathrm{ab}}$ & $10.44 \pm 2.51^{\mathrm{a}}$ & $11.13 \pm 2.12^{\mathrm{a}}$ & $10.18 \pm 1.59^{\mathrm{a}}$ & $10.10 \pm 2.06^{\mathrm{a}}$ \\
\hline Streptococcus ratti & $13.87 \pm 0.69^{\mathrm{a}}$ & $13.27 \pm 0.31^{\mathrm{ab}}$ & $12.34 \pm 0.03^{\mathrm{a}}$ & $13.12 \pm 0.72^{\mathrm{bc}}$ & $13.69 \pm 2.02^{\mathrm{ab}}$ & $15.51 \pm 0.53^{\mathrm{a}}$ & $13.00 \pm 0.32^{\mathrm{b}}$ & $12.89 \pm 0.91^{\mathrm{b}}$ & $11.88 \pm 0.66^{\mathrm{b}}$ & $14.17 \pm 1.06^{\mathrm{a}}$ & $12.75 \pm 0.63^{\text {ab }}$ & $11.82 \pm 0.93^{b}$ \\
\hline $\begin{array}{l}\text { Streptococcus } \\
\text { sanguinis }\end{array}$ & $12.74 \pm 0.78^{\mathrm{ab}}$ & $13.17 \pm 0.74^{\mathrm{a}}$ & $11.98 \pm 1.19^{b}$ & $13.01 \pm 0.57^{\mathrm{a}}$ & $13.08 \pm 1.24^{\mathrm{a}}$ & $14.26 \pm 1.50^{\mathrm{a}}$ & $13.30 \pm 1.63^{\mathrm{a}}$ & $13.27 \pm 0.57^{\mathrm{a}}$ & $13.41 \pm 0.51^{\mathrm{a}}$ & $15.13 \pm 0.98^{\mathrm{a}}$ & $13.55 \pm 1.00^{\mathrm{a}}$ & $13.16 \pm 1.41^{\mathrm{a}}$ \\
\hline $\begin{array}{l}\text { Actinomyces } \\
\text { viscosus }\end{array}$ & $14.72 \pm 2.51^{\mathrm{a}}$ & $14.78 \pm 2.25^{\mathrm{a}}$ & $12.83 \pm 1.44^{\mathrm{a}}$ & $14.59 \pm 1.33^{\mathrm{a}}$ & $13.51 \pm 3.01^{\mathrm{a}}$ & $12.45 \pm 2.77^{\mathrm{a}}$ & $13.43 \pm 2.97^{\mathrm{a}}$ & $14.18 \pm 2.54^{\mathrm{a}}$ & $13.00 \pm 2.68^{\mathrm{a}}$ & $15.19 \pm 2.71^{\mathrm{a}}$ & $13.40 \pm 2.86^{\mathrm{a}}$ & $12.59 \pm 2.63^{\mathrm{a}}$ \\
\hline $\begin{array}{l}\text { Actinomyces } \\
\text { naeslundii }\end{array}$ & $14.34 \pm 0.47^{\mathrm{a}}$ & $14.37 \pm 1.25^{\mathrm{ab}}$ & $13.64 \pm 0.87^{\mathrm{b}}$ & $13.29 \pm 0.13^{\mathrm{b}}$ & $13.86 \pm 0.16^{\mathrm{b}}$ & $15.21 \pm 0.40^{\mathrm{a}}$ & $13.22 \pm 1.04^{\mathrm{b}}$ & $13.34 \pm 0.72^{\mathrm{b}}$ & $12.57 \pm 0.80^{\mathrm{a}}$ & $13.86 \pm 0.93^{\mathrm{a}}$ & $12.39 \pm 1.11^{\mathrm{a}}$ & $12.11 \pm 0.91^{\mathrm{a}}$ \\
\hline $\begin{array}{l}\text { Streptococcus } \\
\text { anginosus }\end{array}$ & $13.54 \pm 2.14^{\mathrm{a}}$ & $14.94 \pm 2.82^{\mathrm{a}}$ & $12.85 \pm 1.90^{\mathrm{a}}$ & $13.18 \pm 2.13^{\mathrm{a}}$ & $13.80 \pm 2.15^{\mathrm{a}}$ & $14.53 \pm 2.57^{\mathrm{a}}$ & $13.81 \pm 2.33^{\mathrm{a}}$ & $13.38 \pm 2.29^{\mathrm{a}}$ & $12.58 \pm 2.55^{\mathrm{a}}$ & $14.42 \pm 2.02^{\mathrm{a}}$ & $13.62 \pm 1.77^{\mathrm{a}}$ & $12.40 \pm 1.77^{\mathrm{a}}$ \\
\hline $\begin{array}{l}\text { Aggregatibacter } \\
\text { actinomycetemcomi } \\
\text { tans }\end{array}$ & $14.34 \pm 0.93^{\mathrm{a}}$ & $14.72 \pm 0.89^{\mathrm{a}}$ & $13.21 \pm 0.91^{\mathrm{b}}$ & $14.26 \pm 0.48^{\mathrm{a}}$ & $14.92 \pm 0.66^{\mathrm{a}}$ & $15.48 \pm 0.53^{\mathrm{a}}$ & $13.80 \pm 1.87^{\mathrm{a}}$ & $14.10 \pm 0.50^{\mathrm{a}}$ & $12.86 \pm 0.83^{b}$ & $15.22 \pm 0.71^{\mathrm{a}}$ & $13.97 \pm 0.94^{\mathrm{ab}}$ & $12.57 \pm 0.45^{\mathrm{b}}$ \\
\hline
\end{tabular}

Values represent means \pm standard deviation, $\mathrm{n}=3$.

Different letters in rows indicate significant differences between mean values of treatments $(p<0.05)$. 
Antimicrobial effect...

YU, S. C. et al.

Table 6. Antimicrobial activities of P. mume extract according to $\mathrm{pH}$.

Diameter of the zone of inhibition (mm, Means \pm SD)

\begin{tabular}{|c|c|c|c|c|c|c|c|c|c|c|c|c|}
\hline \multirow[b]{3}{*}{ Microorganisms } & \multicolumn{12}{|c|}{ Diameter of the zone of inhibition (mm, Means \pm SD) } \\
\hline & \multicolumn{4}{|c|}{ Pectinex Ultra SP-L } & \multicolumn{4}{|c|}{ Novozym 33095} & \multicolumn{4}{|c|}{ Viscozyme L } \\
\hline & $\mathrm{pH} 4$ & pH 5 & $\mathrm{pH} 6$ & $\mathrm{pH} 7$ & $\mathrm{pH} 5$ & $\mathrm{pH} 6$ & $\mathrm{pH} 7$ & $\mathrm{pH} 8$ & $\mathrm{pH} 5$ & $\mathrm{pH} 6$ & $\mathrm{pH} 7$ & $\mathrm{pH} 8$ \\
\hline $\begin{array}{l}\text { Staphylococcus } \\
\text { sobrinus }\end{array}$ & $11.88 \pm 2.25^{\mathrm{a}}$ & $13.76 \pm 1.30^{\mathrm{a}}$ & $14.76 \pm 1.69^{\mathrm{a}}$ & $13.80 \pm 2.01^{\mathrm{a}}$ & $14.58 \pm 2.80^{\mathrm{a}}$ & $14.62 \pm 1.81^{\mathrm{a}}$ & $16.19 \pm 2.99^{\mathrm{a}}$ & $14.56 \pm 3.36^{\mathrm{a}}$ & $14.29 \pm 2.90^{\mathrm{a}}$ & $15.14 \pm 4.03^{\mathrm{a}}$ & $14.80 \pm 3.82^{\mathrm{a}}$ & $14.21 \pm 4.62^{\mathrm{a}}$ \\
\hline $\begin{array}{l}\text { Streptococcus } \\
\text { aureus }\end{array}$ & $12.09 \pm 1.37^{\mathrm{b}}$ & $13.63 \pm 0.99^{\mathrm{ab}}$ & $14.85 \pm 0.90^{\mathrm{a}}$ & $13.92 \pm 0.88^{\mathrm{ab}}$ & $14.31 \pm 2.17^{\mathrm{a}}$ & $14.09 \pm 1.36^{\mathrm{a}}$ & $15.16 \pm 0.91^{\mathrm{a}}$ & $14.04 \pm 1.49^{\mathrm{a}}$ & $13.06 \pm 2.11^{\mathrm{a}}$ & $14.12 \pm 1.70^{\mathrm{a}}$ & $12.60 \pm 1.99^{\mathrm{a}}$ & $11.96 \pm 2.73^{\mathrm{a}}$ \\
\hline $\begin{array}{l}\text { Streptococcus } \\
\text { mutans }\end{array}$ & $10.20 \pm 1.05^{\mathrm{b}}$ & $12.32 \pm 1.43^{\mathrm{a}}$ & $12.39 \pm 0.32^{\mathrm{a}}$ & $11.19 \pm 0.58^{\mathrm{ab}}$ & $14.63 \pm 2.00^{\mathrm{a}}$ & $13.84 \pm 2.30^{\mathrm{a}}$ & $15.68 \pm 1.88^{\mathrm{a}}$ & $14.84 \pm 2.64^{\mathrm{a}}$ & $12.20 \pm 2.08^{\mathrm{a}}$ & $13.28 \pm 2.60^{\mathrm{a}}$ & $11.47 \pm 2.25^{\mathrm{a}}$ & $11.24 \pm 2.33^{\mathrm{a}}$ \\
\hline Streptococcus ratti & $11.93 \pm 1.76^{\mathrm{a}}$ & $14.48 \pm 1.20^{\mathrm{a}}$ & $15.41 \pm 2.07^{\mathrm{a}}$ & $14.22 \pm 2.64^{\mathrm{a}}$ & $13.19 \pm 1.66^{\mathrm{a}}$ & $13.22 \pm 1.42^{\mathrm{a}}$ & $14.85 \pm 3.32^{\mathrm{a}}$ & $13.50 \pm 1.75^{\mathrm{a}}$ & $13.08 \pm 1.52^{\mathrm{a}}$ & $14.64 \pm 2.65^{\mathrm{a}}$ & $12.49 \pm 2.24^{\mathrm{a}}$ & $12.08 \pm 1.76^{\mathrm{a}}$ \\
\hline $\begin{array}{l}\text { Streptococcus } \\
\text { sanguinis }\end{array}$ & $12.89 \pm 3.80^{\mathrm{a}}$ & $14.10 \pm 3.92^{\mathrm{a}}$ & $15.28 \pm 4.33^{\mathrm{a}}$ & $14.61 \pm 4.17^{\mathrm{a}}$ & $14.79 \pm 3.99^{\mathrm{a}}$ & $13.70 \pm 3.67^{\mathrm{a}}$ & $15.04 \pm 4.81^{\mathrm{a}}$ & $13.64 \pm 5.05^{\mathrm{a}}$ & $13.97 \pm 4.76^{\mathrm{a}}$ & $14.95 \pm 4.80^{\mathrm{a}}$ & $13.99 \pm 4.76^{\mathrm{a}}$ & $13.02 \pm 5.00^{\mathrm{a}}$ \\
\hline $\begin{array}{l}\text { Actinomyces } \\
\text { viscosus }\end{array}$ & $10.07 \pm 0.19^{\mathrm{b}}$ & $12.22 \pm 1.10^{\mathrm{ab}}$ & $13.00 \pm 1.90^{\mathrm{a}}$ & $11.42 \pm 0.78^{\mathrm{ab}}$ & $12.92 \pm 1.53^{\mathrm{a}}$ & $12.98 \pm 1.28^{\mathrm{a}}$ & $15.46 \pm 1.37^{\mathrm{a}}$ & $13.52 \pm 1.68^{\mathrm{a}}$ & $13.06 \pm 2.28^{\mathrm{a}}$ & $14.28 \pm 2.31^{\mathrm{a}}$ & $12.08 \pm 1.34^{\mathrm{a}}$ & $11.59 \pm 2.28^{\mathrm{a}}$ \\
\hline $\begin{array}{l}\text { Actinomyces } \\
\text { naeslundii }\end{array}$ & $11.89 \pm 2.60^{\mathrm{a}}$ & $13.81 \pm 2.61^{\mathrm{a}}$ & $15.41 \pm 1.49^{\mathrm{a}}$ & $13.94 \pm 2.15^{\mathrm{a}}$ & $14.65 \pm 1.97^{\mathrm{a}}$ & $14.73 \pm 1.82^{\mathrm{a}}$ & $16.53 \pm 1.66^{\mathrm{a}}$ & $15.32 \pm 2.40^{\mathrm{a}}$ & $15.52 \pm 2.10^{\mathrm{a}}$ & $15.97 \pm 2.63^{\mathrm{a}}$ & $14.66 \pm 2.72^{\mathrm{a}}$ & $14.04 \pm 2.31^{\mathrm{a}}$ \\
\hline $\begin{array}{l}\text { Streptococcus } \\
\text { anginosus }\end{array}$ & $12.42 \pm 2.93^{\mathrm{a}}$ & $14.03 \pm 3.11^{\mathrm{a}}$ & $14.72 \pm 2.88^{\mathrm{a}}$ & $13.28 \pm 3.51^{\mathrm{a}}$ & $13.14 \pm 2.43^{\mathrm{a}}$ & $13.04 \pm 2.04^{\mathrm{a}}$ & $13.76 \pm 2.61^{\mathrm{a}}$ & $12.94 \pm 1.71^{\mathrm{a}}$ & $14.55 \pm 2.67^{\mathrm{a}}$ & $15.47 \pm 3.76^{\mathrm{a}}$ & $13.50 \pm 1.28^{\mathrm{a}}$ & $12.88 \pm 2.48^{\mathrm{a}}$ \\
\hline $\begin{array}{l}\text { Aggregatibacter } \\
\text { actinomycetemcomi } \\
\text { tans }\end{array}$ & $12.13 \pm 2.43^{\mathrm{a}}$ & $13.78 \pm 3.01^{\mathrm{a}}$ & $15.58 \pm 1.34^{\mathrm{a}}$ & $13.68 \pm 2.02^{\mathrm{a}}$ & $14.17 \pm 3.30^{\mathrm{a}}$ & $15.40 \pm 2.31^{\mathrm{a}}$ & $15.85 \pm 2.41^{\mathrm{a}}$ & $15.05 \pm 2.51^{\mathrm{a}}$ & $15.14 \pm 3.65^{\mathrm{a}}$ & $16.14 \pm 3.76^{\mathrm{a}}$ & $14.12 \pm 4.07^{\mathrm{a}}$ & $13.07 \pm 3.33^{\mathrm{a}}$ \\
\hline
\end{tabular}

Values represent means \pm standard deviation, $\mathrm{n}=3$.

Different letters in rows indicate significant differences between mean values of treatments $(p<0.05)$. 

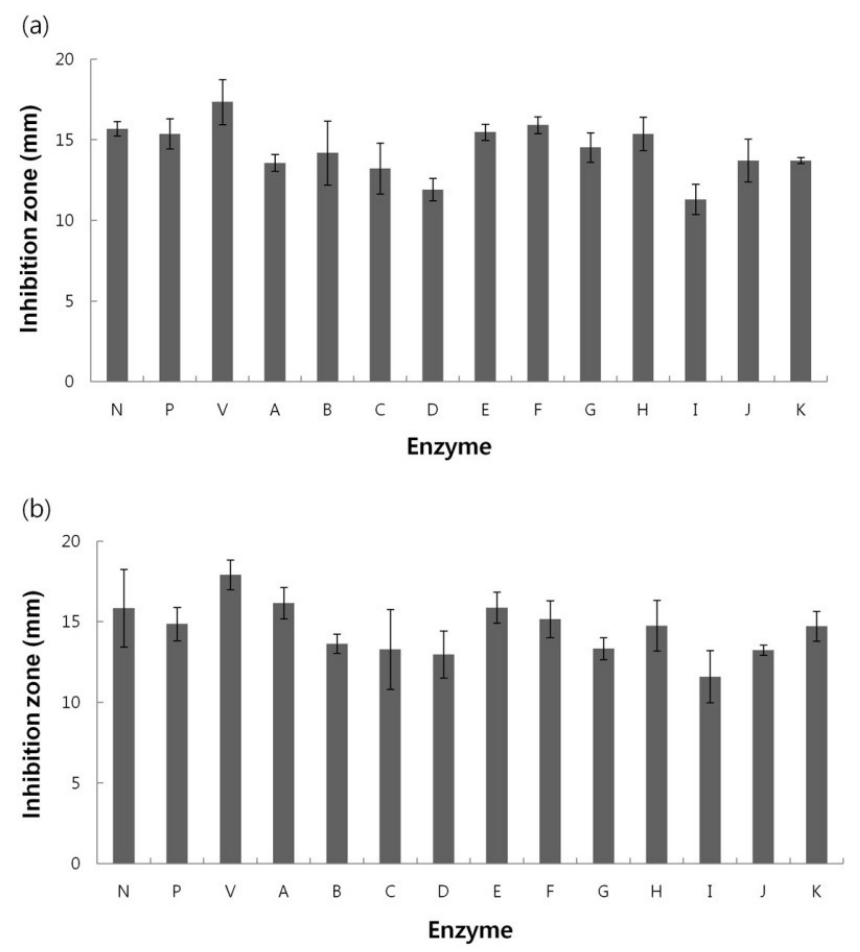

Figure 2. Antimicrobial activities of $P$. mume extracted using combinations of enzymes a) Actinomyces naeslundii and b) Aggregatibacter actinomycetemcomitans. Bars are means \pm standard deviation, $\mathrm{n}=3$. N: Novozym 33095; P: Pectinex Ultra SP-L; V: Viscozyme L; A: combination of Novozym and Viscozyme, ratio of Novozym and Viscozyme $=8: 2 ;$ B: combination of Novozym and Viscozyme, ratio of Novozym to Viscozyme $=6: 4$; : combination of Novozym and Viscozyme, ratio of Novozym to Viscozyme $=4: 6$; D: combination of Novozym and Viscozyme, ratio of Novozym to Viscozyme $=2: 8$; E: combination of Novozym and Pectinex, ratio of Novozym to Pectinex $=8: 2$; F: combination of Novozym and Pectinex, ratio of Novozym to Pectinex $=6: 4 ;$ G: combination of Novozym and Pectinex, ratio of Novozym to Pectinex $=4: 6$; H: combination of Novozym and Pectinex, ratio of Novozym to Pectinex $=2: 8$; I: combination of Viscozyme, Pectinex and Novozym, ratio of Viscozyme, Pectinex and Novozym $=6: 2: 2 ; \mathrm{J}$ : combination of Viscozyme, Pectinex and Novozym, ratio of Viscozyme, Pectinex and Novozym $=2: 6: 2$; K: combination of Viscozyme, Pectinex and Novozym, ratio of Viscozyme, Pectinex and Novozym $=2: 2: 6$

\section{Determination of MIC}

The optimal conditions for enzymatic extraction were $\mathrm{pH} 6.0,45^{\circ} \mathrm{C}$, and $20 \mathrm{~h}$ for Pectinex Ultra SP-L. The maximum antimicrobial activity of $P$. mume extract by Novozym 33095 was obtained at $\mathrm{pH} 7.0,45^{\circ} \mathrm{C}$, and $20 \mathrm{~h}$. The Viscozyme L extract showed the greatest inhibitory effect at $\mathrm{pH}$ 6.0, $45^{\circ} \mathrm{C}$, and $20 \mathrm{~h}$. The MICs of $P$. mume extracts against oral bacterial strains were determined using a broth microdilution method. The MIC was defined as the concentration at which a sharp decline, followed by a constant absorbance value, occurred. The MICs of $P$. mume extracts under the optimum conditions are presented in Table 7. The concentration of extract was expressed as a percentage dilution of the original extract of $P$. mume. The extracts exhibited MIC values of 3.125 to $12.50 \%$ against the bacteria species. The Pectinex Ultra SP-L extract showed a MIC of $6.25 \%$ against S. mutans, S. sanguinis, S. anginosus, A. viscosus, and $A$. naeslundii. The Novozym 33095 extract exhibited an MIC of $6.25 \%$ against $S$. sanguinis, $A$. viscosus, $A$. naeslundii, and $A$. actinomycetemcomitans. The Viscozyme L extract exhibited an MIC of $6.25 \%$ against $S$. sobrinus, $S$. mutans, S. anginosus, A. viscosus, and A. naeslundii. $S$. sanguinis showed the greatest susceptibility (MIC, 3.125\%). Indeed, all extracts showed higher antimicrobial activity against $S$. sanguinis, than the other bacterial strains. The MIC of enzyme combination extracts did not differ significantly compared with those of each enzyme alone. 
Table 7. Minimum inhibitory concentrations (\% of dilution) of P. mume extracts.

\begin{tabular}{lccccccc}
\hline & \multicolumn{7}{c}{ MIC (\% of dilution) } \\
\cline { 2 - 8 } Microorganisms & \multicolumn{7}{c}{ Enzyme } \\
\cline { 2 - 8 } & $\mathrm{N}$ & $\mathrm{P}$ & $\mathrm{V}$ & $\mathrm{C} 1$ & $\mathrm{C} 2$ & $\mathrm{C} 3$ & $\mathrm{C} 4$ \\
\hline S. aureus & 12.50 & 12.50 & 6.25 & 12.50 & 12.50 & 12.50 & 12.50 \\
Staphylococcus sobrinus & 12.50 & 12.50 & 6.25 & 3.125 & 12.50 & 12.50 & 12.50 \\
S. mutans & 12.50 & 6.25 & 6.25 & 6.25 & 6.25 & 12.50 & 6.25 \\
S. ratti & 12.50 & 12.50 & 6.25 & 12.50 & 6.25 & 12.50 & 12.50 \\
S. sanguinis & 6.25 & 6.25 & 3.125 & 6.25 & 6.25 & 6.25 & 6.25 \\
S. anginosus & 12.50 & 6.25 & 6.25 & 12.50 & 12.50 & 12.50 & 12.50 \\
A. viscosus & 6.25 & 6.25 & 6.25 & 6.25 & 6.25 & 6.25 & 12.50 \\
A. naeslundii & 6.25 & 6.25 & 6.25 & 6.25 & 6.25 & 12.50 & 12.50 \\
Aggregatibacter & 6.25 & 12.50 & 12.50 & 6.25 & 6.25 & 12.50 & 12.50 \\
actinomycetemcomitans & & & & & & \\
\hline
\end{tabular}

Values represent the percentages of dilution on the basis of the original extract.

N: Novozym 33095; P: Pectinex Ultra SP-L; V: Viscozyme L; C1: combination of Novozym and Pectinex, ratio of Novozym to Pectinex $=8: 2 ;$ C2: combination of Novozym and Viscozyme, ratio of Novozym to Viscozyme $=8: 2$; C3: combination of Pectinex and Novozym, ratio of Pectinex to Novozym = 8:2; C4: combination of Pectinex and Viscozyme, ratio of Pectinex to Viscozyme $=8: 2$.

Dental caries and periodontal disease are the most important infectious oral diseases. Oral diseases can be prevented using antimicrobial agents such as chlorhexidine that alter the oral microbiota and result in teeth staining (WALSH et al., 2015). Therefore, alternative natural products have been investigated, with a focus on and phytochemical antibacterial compounds (GONZALEZ et al., 2013). Previous observations have suggested that $P$. mume solvent extract exhibits antioxidant and antimicrobial activities (PINELO et al., 2006; XIA et al., 2011). Unfortunately, these methods suffer from low extraction yields, long extraction times and potential for trace organic solvents in the final products, which decreases the product quality $(\mathrm{CHO}$ et al., 2013).

High-pressure enzymatic extraction of phytochemical compounds is a potential alternative to solvent-based extraction. In this study, enzymatic extraction was conducted at $100 \mathrm{MPa}$, which does not affect the stability and functionality of most enzymes (MASSON et al., 2001; SUNWOO et al., 2013). The efficiency of enzymatic extraction is influenced by multiple variables, including enzyme concentration, incubation time, incubation temperature, $\mathrm{pH}$ and use of enzyme combinations (LANDBO; MEYER, 2001; PINELO et al., 2006). Accordingly, the high-pressure enzymatic extraction conditions should be optimized to maximize the antimicrobial activity against oral bacteria. We found that enzymatic treatment increased the inhibitory effects significantly in comparison to the control treatment. Briefly, the P. mume cell wall was disrupted by treatment with Pectinex Ultra SP-L,
Novozym 33095 and Viscozyme L, which resulted in the release of biologically active components. With enzymatic treatment, the longer the incubation time, the greater the hydrolysis of cell wall components. However, a longer hydrolysis time will result in increased microbial growth.

The polyphenols of some edible plants have been reported as potential sources of agents capable of controlling the growth of oral bacteria (YOO et al., 2011). Temperature has the greatest influence on the release of phenols from phytochemical compounds (MEYER et al., 1998). With increasing temperature, antimicrobial activity increases due to enhanced solubility, enzyme activity, and diffusion coefficient. However, a temperature $>45^{\circ} \mathrm{C}$ affects activity negatively because of the instability of the phenolic compounds and membrane denaturation (PINELO et al., 2006). A temperature higher or lower than $45^{\circ} \mathrm{C}$ partly inhibited enzyme activity as reported previously (LANDBO; MEYER, 2001). The maximum inhibition zone diameter was obtained at pH 6.0 or 7.0, depending on the enzyme. The $\mathrm{pH}$ value of the reaction solution plays an important role in cell wall hydrolysis and polyphenol extraction in plants (ZHENG et al., 2008). Use of combinations of enzymes did not significantly affect activity compared with each enzyme alone. Especially, used of Viscozyme L led to a decrease in the antimicrobial activities against $A$. naeslundii and $A$. actinomycetemcomitans. The $P$. mume extracts showed similar MIC values irrespective of the enzyme used. However, Viscozyme L showed lower MIC values than other enzymes for all oral bacteria tested. 
The antimicrobial activities of $P$. mume extracts obtained by solvent extraction have been reported that they inhibited oral bacterial strains, such as $S$. mutans, $S$. sobrinus, $S$. gingivalis, and $A$. actinomycetemcomitans (SENEVIRATNE et al., 2011; JANG et al., 2014). However, it has not been investigated about the antimicrobial activities of $P$. mume extracts based on high-pressure enzymatic treatment against oral bacteria. In this study, $P$. mume extracts obtained by high-pressure enzymatic treatment exhibited antimicrobial activity superior to that reported in the above-mentioned study. Thus, high-pressure enzymatic extraction yields products with considerable antimicrobial activity.

\section{CONCLUSIONS}

High-pressure enzymatic treatment induces release of biologically active components from the cell wall matrix, which results in enhanced antimicrobial activity. We determined the optimum $\mathrm{pH}$, temperature, incubation time, and enzyme concentration for the extraction of $P$. mume using three commercially available enzymes (Pectinex Ultra SP-L, Novozym 33095 and Viscozyme L) in a high-pressure (100 $\mathrm{MPa})$ reactor.

The optimal conditions were $5 \mathrm{v} / \mathrm{v} \%$ enzyme concentration at a temperature of $45^{\circ} \mathrm{C}$, an incubation time of $20 \mathrm{~h}$, at $\mathrm{pH} 6.0$ or $7.0(\mathrm{pH} 7.0$ with Novozym 33095, pH 6.0 with Pectinex Ultra SP-L or Viscozyme L).
The $P$. mume extracted by high-pressure enzymatic extraction have higher antimicrobial activity compared to that of conventional solvent extraction. Therefore, compared with a solvent extraction method using large quantity of organic solvent, the high-pressure enzymatic extraction is a novel and environmentally friendly approach for extraction of ingredients with antimicrobial activity against oral bacteria.

The pharmaceutical and food industry can be promoted by the use of high-pressure enzymatic extraction, which provides high extraction efficiency with short extraction time, fewer impurities, and non-solvent use.

\section{ACKNOWLEDGMENTS}

This research was supported by Basic Science Research Program through the National Research Foundation of Korea (NRF) funded by the Ministry of Science, ICT \& Future Planning (2017R1A2B4012865) and was supported by Business for Cooperative R\&D between Industry, Academy, and Research Institute funded Korea Small and Medium Business Administration in 2014 (C0190937). In addition, this work was carried out with the support of "Cooperative Research Program for National Agricultural Genome Program (Project No. PJ01337303)" Rural Development Administration, Republic of Korea.

RESUMO: Avaliar o processamento de alta pressão combinado com o tratamento enzimático para a extração de Prunus mume, determinando as condições ótimas de extração para a atividade antimicrobiana contra as bactérias orais. A extração enzimática de alta pressão foi utilizada para isolar os componentes biologicamente ativos de $P$. mume. Os efeitos das variáveis do processo como o tipo enzimático (Pectinex Ultra SP-L, Novozym 33095 e Viscozyme L), a concentração de enzima, o tempo/temperatura de incubação, $\mathrm{pH}$, e a relação da atividade antimicrobiana de enzimas contra os patógenos orais relacionados à cárie dentária e doenças periodontais foram determinados pelo ensaio de difusão em disco. As condições ótimas para a extração enzimática de $P$. mume foram $\mathrm{pH} 6.0,45^{\circ} \mathrm{C}, 20 \mathrm{~h}$, e $5 \mathrm{v} / \mathrm{v} \%$ com Pectinex Ultra SP-L. A máxima atividade antimicrobiana do extrato de $P$. mume obtida usando Novozym 33095 foi em $\mathrm{pH} 7.0,45^{\circ} \mathrm{C}, 20 \mathrm{~h}$, e 5 v/v\%. O extrato de Viscozyme L apresentou o efeito inibitório máximo em $\mathrm{pH} 6.0,45^{\circ} \mathrm{C}, 20 \mathrm{~h}$, e $5 \mathrm{v} / \mathrm{v} \%$. O uso de combinações de enzimas não resultou em uma atividade antimicrobiana significativamente diferente $(\mathrm{p}<0.05)$ em comparação com cada enzima por separada. Os valores mínimos da concentração inibitória foram de 3.125 a $12.50 \%$. Estes resultados indicaram que a extração enzimática de alta pressão produziu o extrato de $P$. mume com atividade antimicrobiana,o qual tem o potencial para melhorar o ambiente bucal.

PALAVRAS-CHAVE: Atividade antimicrobiana. Extrato enzimático de alta pressão. Concentração Inibitória Mínima. Prunus mume.

\section{REFERENCES}

BOUAYED, J.; RAMMAL, H.; DICKO, A.; YOUNOS, C.; SOULIMANI, R. The antioxidant effect of plums polyphenolic compounds against $\mathrm{H}\left({ }_{2}\right) \mathrm{O}(2)$-induced oxidative stress in mouse blood granulocytes. Journal of Medicinal Food, v. 12, n. 4, p. 861-868, 2009. http://dx.doi.org/10.1089/jmf.2008.0165 
CHO, J. H.; BHATTARAI, S.; OH, T. J.; JANG, J. H. Enzymatic extraction of pilocarpine from Pilocarpus jaborandi. Korean Journal of Microbiology and Biotechnology, v. 41, n. 2, p. 236-241, 2013. http://dx.doi.org/10.4014/kjmb.1303.03005

GONZALEZ, O. A.; ESCAMILLA, C.; DANAHER, R. J.; DAI, J.; EBERSOLE, J. L.; MUMPER, R. J. Antibacterial effects of blackberry extract target periodontopathogens. Journal of Periodontal Research, v. 48, n. 1, p. 80-86, 2013. http://dx.doi.org/10.1111/j.1600-0765.2012.01506

JANG, J. H.; KIM, Y. I.; LEE, H. Antimicrobial activity of Prunus mume extract to oral microbes. Journal of Korean Society of Dental Hygiene, v. 14, n. 1, p. 109-115, 2014.

http://dx.doi.org/10.13065/jksdh.2014.14.01.109

JUN, X.; DEJI, S.; YE, L.; RUI, Z. Comparison of in vitro antioxidant activities and bioactive components of green tea extracts by different extraction methods. International Journal of Pharmaceutics, v. 408, n. 1-2, p. 97-101, 2011. http://dx.doi.org/10.1016/j.ijpharm.2011.02.002

KAWAHARA, K.; HASHIGUCHI, T.; MASUDA, K.; SANIABADI, A. R.; KIKUCHI, K.; TANCHAROEN, S. ITO, T.; MIURA, N.; MORIMOTO, Y.; BISWAS, K. K.; NAWA, Y.; MENG, X.; OYAMA, Y.; TAKENOUCHI, K.; SHRESTHA, B.; SAMESHIMA, H.; SHIMIZU, T.; ADACHI, T.; ADACHI, M.; MARUYAMA, I. Mechanism of HMGB1 release inhibition from RAW264.7 cells by oleanolic acid in Prunus mume Sieb. et Zucc. International Journal of Molecular Medicine, v. 23, n. 5, p. 615-620, 2009.

LANDBO, A. K.; MEYER, A. S. Enzyme-assisted extraction of antioxidative phenols from black currant juice press residues (Rives nigrum). Journal of Agricultural and Food Chemistry, v. 49, n. 7, p. 3169-3177, 2001.

LIEN, H. M.; TSENG, C. J.; HUANG, C. L.; LIN, Y. T.; CHEN, C. C.; LAI, Y. Y. Antimicrobial activity of Antrodia camphorata extracts against oral bacteria. PLoS One. v. 9, n. 8, p. e105286, 2014. http://dx.doi.org/10.1371/journal.pone.0105286

MASSON, P.; TONELLO, C.; BALNY, C. High-pressure biotechnology in medicine and pharmaceutical science. Journal of Biomedicine and Biotechnology, v. 1, n. 2, p. 85-88, 2001.

http://dx.doi.org/10.1155/S1110724301000158

MEYER, A. S.; JEPSEN, S. M.; SORENSEN, N. S. Enzymatic release of antioxidants for human low-density lipoprotein from grape pomace. Journal of Agricultural and Food Chemistry, v. 46, n. 7, p. 2439-2446, 1998. http://dx.doi.org/10.1021/jf971012f

PIDDOCK, L. J. Techniques used for the determination of antimicrobial resistance and sensitivity in bacteria. Antimicrobial Agents Research Group. Journal of Applied Bacteriology, v. 68, n. 4, p. 307-318, 1990.

PINELO, M.; ARNOUS, A.; MEYER, A. S. Upgrading of grape skins: significance of plant cell-wall structural components and extraction techniques for phenol release. Trends in Food Science and Technology, v. 17, n. 11, p. 579-590, 2006. http://dx.doi.org/10.1016/j.tifs.2006.05.003

SENEVIRATNE, C. J.; WONG, R. W.; HAGG, U.; CHEN, Y.; HERATH, T. D.; SAMARANAYAKE, P. L.; KAO, R. Prunus mume extract exhibits antimicrobial activity against pathogenic oral bacteria. International Journal of Paediatric Dentistry, v. 21, n. 4, p. 299-305, 2011. http://dx.doi.org/10.1111/j.1365-

263X.2011.01123

SOCRANSKY, S. S.; HAFFAJEE, A. D. The bacterial etiology of destructive periodontal desease: current concepts. Journal of Periodontology, v. 63, n. 4 Suppl, p. 322-331, 1992.

http://dx.doi.org/10.1902/jop.1992.63.4s.322 
SUNWOO, H. H.; KIM, C. T.; KIM, D. Y.; MAENG, J. S.; CHO, C. W.; LEE, S. J. Extraction of ginsenosides from fresh ginseng roots (Panax ginseng C.A. Meyer) using commercial enzymes and high hydrostatic pressure. Biotechnology Letters, v. 35, n. 7, p. 1017-1022, 2013. http://dx.doi.org/10.1007/s10529-013-1182

SWENSON, J. M.; THORNSBERRY, C.; SILCOX, V. A. Rapidly growing mycobacteria: testing of susceptibility to 34 antimicrobial agents by microdilution. Antimicrobial Agents and Chemotherapy, v. 22, n. 2, p. 186-192, 1982.

VAN HOUTE, J. Role of micro-organisms in caries etiology. Journal of Dental Research, v. 73, n. 3, p. 672681, 1994. http://dx.doi.org/10.1177/00220345940730031301

WALSH, T.; OLIVEIRA-NETO, J. M.; MOORE, D. Chlorhexidine treatment for the prevention of dental caries in children and adolescents. The Cochrane Database of Systematic Reviews, v. 4, p. CD008457, 2015. http://dx.doi.org/10.1002/14651858.CD008457.pub2

XIA, D.; WU, X.; SHI, J.; YANG, Q.; ZHANG, Y. Phenolic compounds from the edible seeds extract of Chinese Mei (Prunus mume Sieb. et Zucc) and their antimicrobial activity. LWT - Food Science and Technology, v. 44, n. 1, p. 347-349, 2011. http://dx.doi.org/10.1016/j.lwt.2010.05.017

YOO, S.; MURATA, R. M.; DUARTE, S. Antimicrobial traits of tea- and cranberry-derived polyphenols against Streptococcus mutans. Caries Research, v. 45, n. 4, p. 327-335, 2011.

http://dx.doi.org/10.1159/000329181

ZHENG, H. Z.; LEE, H. R.; LEE, S. H.; KIM, C. S.; CHUNG, S. K. Pectinase assisted extraction of polyphenol from apple pomace. Chinese Journal of Analytical Chemistry, v. 36, n. 3, p. 306-310, 2008. 